BULLETIN (New Series) OF THE

AMERICAN MATHEMATICAL SOCIETY

Volume 29, Number 1, July 1993

\title{
A COUNTEREXAMPLE TO BORSUK'S CONJECTURE
}

\author{
JEFF KAHN AND GIL KALAI
}

\begin{abstract}
Let $f(d)$ be the smallest number so that every set in $R^{d}$ of diameter 1 can be partitioned into $f(d)$ sets of diameter smaller than 1 . Borsuk's conjecture was that $f(d)=d+1$. We prove that $f(d) \geq(1.2)^{\sqrt{d}}$ for large $d$.
\end{abstract}

\section{INTRODUCTION}

Sixty years ago Borsuk [2] raised the following question.

Problem 1 (Borsuk). Is it true that every set of diameter one in $R^{d}$ can be partitioned into $d+1$ closed sets of diameter smaller than one? The conjecture that this is true has come to be called Borsuk's conjecture.

Let $f(d)$ be the smallest number so that every set in $R^{d}$ of diameter 1 can be partitioned into $f(d)$ sets of diameter smaller than 1 . The vertices of the regular simplex in $R^{d}$ show that $f(d) \geq d+1$. (Another example showing this is, by the Borsuk-Ulam theorem, the $d$-dimensional Euclidean ball.) The assertion of Borsuk's conjecture was proved in dimensions 2 and 3 and in all dimensions for centrally symmetric convex bodies and smooth convex bodies. See $[9,1,4]$ and references cited there. Lassak [14] proved that $f(d) \leq 2^{d-1}+1$, and Schramm [16] showed that for every $\epsilon$, if $d$ is sufficiently large, $f(d) \leq(\sqrt{(3 / 2)}+\epsilon)^{d}$. A different proof of Schramm's bound was given by Bourgain and Lindenstrauss [3]. See [9, 1, 4] for surveys and many references on Borsuk's problem.

Borsuk's conjecture seems to have been believed generally, and various stronger conjectures have been proposed. The possibility of a counterexample based on combinatorial configurations was suggested by Erdős [6], Larman [15], and perhaps others. In 1965 Danzer [5] showed that the finite set $K \subseteq R^{d}$ consisting of all $\{0,1\}$-vectors of an appropriate weight cannot be covered by $(1.003)^{d}$ balls of smaller diameter. Larman [13] observed that, for sets consisting of 0-1 vectors with constant weight, Borsuk's conjecture reduces to:

Conjecture 1. Let $K$ be a family of $k$-subsets of $\{1,2, \ldots, n\}$ such that every two members of $K$ have $t$ elements in common. Then $K$ can be partitioned into $n$ parts so that in each part every two members have $(t+1)$ elements in common.

Here we prove

Theorem 1. For large enough $d, f(d) \geq(1.2)^{\sqrt{d}}$ by constructing an appropriate family of sets.

Received by the editors June 30, 1992.

1991 Mathematics Subject Classification. Primary 52A20; Secondary 05D05, 52C17.

The first author was supported in part by BSF, NSF, and AFOSR. The second author was supported in part by GIF. 
We need the following result of Frankl and Wilson [8].

Theorem 2 (Frankl and Wilson). Let $k$ be a prime power and $n=4 k$. Let $K$ be a family of $n / 2$-subsets of $\{1,2, \ldots, n\}$, so that no two sets in the family have intersection of size $n / 4$. Then

$$
|K| \leq 2 \cdot\left(\begin{array}{c}
n-1 \\
n / 4-1
\end{array}\right)
$$

This settled, in particular, a (much weaker) conjecture of Larman and Rogers [12] and implies that, if $g(d)$ is the smallest number so that $R^{d}$ can be colored by $g(d)$ colors such that no two points of the same color are distance one apart, then $g(d) \geq(1.2)^{d}$.

Let us also mention the following related result conjectured by Erdös and proved by Frankl and Rödl [7].

Theorem 3 (Frankl and Rödl). Let $n$ be a positive integer divisible by four. Let $K$ be a family of $n / 2$-subsets of $\{1,2, \ldots, n\}$ such that no two sets in the family have intersection of size $n / 4$. Then $|K| \leq(1.99)^{n}$.

\section{THE CONSTRUCTION}

However contracted, that definition is the result of expanded meditation. -Herman Melville, Moby Dick

Let $V=\{1,2, \ldots, m\}$, and $m=4 k$, and $k$ is a prime power. Let $W$ be the set of pairs of elements in $V$. For every partition $P=\{A, B\}$ of $V$ let $S(A, B)$ be the sets of all pairs which contain one element from $A$ and one element from $B$. Let $K$ be the family of all sets of pairs which correspond to partitions of $V$ into two equal parts, i.e., $K=\{S(A, B):|A|=2 k\}$. Thus, $K$ is a family of $\left(m^{2} / 4\right)$-subsets of an $m(m-1) / 2$-set. The smallest intersection between $S(A, B)$ and $S(C, D)$ occurs when $|A \cap C|=k$, and by the FranklWilson theorem every subfamily of more than $2 \cdot\left(\begin{array}{c}m-1 \\ m / 4-1\end{array}\right)$ sets in $K$ contains two sets which realize the minimal distance. Thus, $K$ cannot be partitioned into fewer than

$$
\frac{\frac{1}{2}\left(\begin{array}{c}
m \\
m / 2
\end{array}\right)}{2 \cdot\left(\begin{array}{c}
m-1 \\
m / 4-1
\end{array}\right)}
$$

parts so that the minimal intersection is not realized in any of the parts. This expression is greater than $(1.203)^{\sqrt{d}}$ for sufficiently large $d=\left(\begin{array}{c}m \\ 2\end{array}\right)-1$, and Theorem 1 for general (large) $d$ follows via the prime number theorem.

\section{REMARKS}

1. In view of Theorem 1, the upper bounds on $f(d)$ cited earlier seem much more reasonable than formerly. It would be of considerable interest to have a better understanding of the asymptotic behavior of $\log f(d)$. At the moment, we cannot distinguish the asymptotic behavior of $f(d)$ from that of $g(d)$. Also of interest would be counterexamples in small dimensions. Our construction shows that Borsuk's conjecture is false for $d=1,325$ and for every $d>2,014$. 
2. Larman's conjecture for $t=1$ is open and still quite interesting, in part because of its similarity to the Erdős-Faber-Lovász conjecture. See [11, 10] for some discussion and related results.

3. Intersection properties of edge-sets of graphs were first studied by Sós; see [17] and references quoted therein.

\section{REFERENCES}

1. V. Boltjansky and I. Gohberg, Results and problems in combinatorial geometry, Cambridge Univ. Press, Cambridge, 1985.

2. K. Borsuk, Drei Sätze über die n-dimensionale euklidische Sphäre, Fund. Math. 20 (1933), 177-190.

3. J. Bourgain and J. Lindenstrauss, On covering a set in $R^{d}$ by balls of the same diameter, Geometric Aspects of Functional Analysis (J. Lindenstrauss and V. Milman, eds.), Lecture Notes in Math., vol. 1469, Springer-Verlag, Berlin, 1991, pp. 138-144.

4. H. Croft, K. Falconer, and R. Guy, Unsolved problems in geometry, Springer-Verlag, New York, 1991, pp. 123-125.

5. L. Danzer, On the $k$-th diameter in $E^{d}$ and a problem of Grünbaum, Proc. Colloq. on Convexity 1965 (W. Fenchel, ed.), Københavns Univ. Math. Inst., 1967.

6. P. Erdös, My Scottish book "problems", The Scottish Book, Mathematics from the Scottish Café (R. D. Mauldin, ed.), Birkhäuser, 1981, pp. 35-43.

7. P. Frankl and V. Rödl, Forbidden intersections, Trans. Amer. Math. Soc. 300 (1987), 259286.

8. P. Frankl and R. Wilson, Intersection theorems with geometric consequences, Combinatorica 1 (1981), 357-368.

9. B. Grünbaum, Borsuk's problem and related questions, Proc. Sympos. Pure Math., vol. 7, Amer. Math. Soc, Providence, RI, 1963.

10. J. Kahn and G. Kalai, A problem of Füredi and Seymour on covering intersecting families by pairs (to appear).

11. J. Kahn and P. Seymour, A fractional version of the Erdös-Faber-Lovász conjecture, Combinatorica 12 (1992), 155-160.

12. D. Larman and C. Rogers, The realization of distances within sets in Euclidean space, Mathematika 19 (1972), 1-24.

13. D. Larman, Open problem 6, Convexity and Graph Theory (M. Rozenfeld and J. Zaks, eds.), Ann. Discrete Math., vol. 20, North-Holland, Amsterdam and New York, 1984, p. 336.

14. M. Lassak, An estimate concerning Borsuk's partition problem, Bull. Acad. Polon. Sci. Ser. Math. 30 (1982),449-451.

15. C. A. Rogers, Some problems in the geometry of convex bodies, The Geometric Vein-The Coxeter Festschrift (C. Davis, B. Grünbaum, and F. A. Sherk, eds.), Springer-Verlag, New York, 1981, pp. 279-284.

16. O. Schramm, Illuminating sets of constant width, Mathematika 35 (1988), 180-199.

17. M. Simonovits and V. Sós, Graph intersection theorems. II, Combinatorics (A. Hajnal and V. Sós, eds.), North-Holland, Amsterdam, 1978, pp. 1017-1030.

Department of Mathematics, Rutgers University, New Brunswick, New Jersey 08903 E-mail address: jkahn@math.rutgers.edu

Institute of Mathematics, Hebrew University, Jerusalem 91904, IsRael

E-mail address: kalai\%humus.huji.ac.il@relay.cs.net 